\section{Integrierte Analyse von Patienten-Genen und respiratorischem Mikrobiom}

Molyneaux PL et al. Host-Microbial interactions in Idiopathic Pulmonary Fibrosis. Am J Resp Crit Care Med 2017; DOI 10.1164/rccm.201607$14080 \mathrm{C}$

Die Ursache der idiopathischen Lungenfibrose (IPF) ist unbekannt. Frühere Studien wiesen Veränderungen des respiratorischen Mikrobioms und genetische Aberrationen nach. Die Zusammenhänge zwischen DNARNA-Transkriptomen, bakterieller Besiedlung und klinischem Verlauf sind unklar. Die Autoren untersuchten die Interaktion mit einer kombinierten Netzwerkanalyse, in die das individuelle Genom, die mikrobielle Signatur und klinische Faktoren einflossen.

Der aktuelle Forschungsstand zur Ätiologie der IPF geht von rezidivierenden Alveolarschädigungen aus, die bei genetisch prädisponierten Patienten mit insuffizienten alveolären Reparaturmechanismen zu Vernarbungen führen. Patienten mit Polymorphismen des TOLLIPund MUC5B-Gens hatten eine erhöhte Vulnerabilität für eine IPF. TOLLIP (Toll interacting protein) stört die Erkennung mikrobieller Pathogene und schwächt die antiinflammatorische Immunantwort. MUC5B ist essenziell für die Makrophagenfunktion und die mukoziliare Clearance von Bakterien. In der prospektiven Studie erhielten 60 Patienten mit einer IPF eine Genanalyse unter Berücksichtigung des TOLLIP- und MUC5B-Genotyps, eine Bronchoskopie mit bronchoalveolärer Lavage (BAL) und eine Lungenfunktion. In 30 Fällen kamen Langzeitdaten hinzu. 20 gesunde Personen bildeten die Kontrollgruppe.

Die Patienten waren durchschnittlich 67,8 Jahre alt und überwiegend männlich (65\%). In den meisten Fällen lag eine mittelschwere Erkrankung vor (Kohlenmonoxid-Diffusionskapazität 40,9\%/ forcierte Vitalkapazität 73,4\%). In 12 Monaten starben 24 Patienten und bei
13 weiteren trat eine Progression ein. Verglichen mit den Kontrollen war die mikrobielle Last bei einer IPF um das 2Fache gesteigert. Patienten mit IPF hatten signifikant mehr DNA-Abschnitte von 4 Spezies (Hämophilus, Neisserien, Streptokokken und Veillonellen). Die integrierte Netzwerkanalyse ergab 2 Module, die mit der Diagnose, der Bakterienlast, den spezifischen operativen taxonomischen Einheiten sowie einer Granulozytose in Blut und BAL assoziiert waren. In den Modulen stachen 6 Gene heraus, die bedeutsame Proteine für die Abwehr von Bakterien kodieren:

- NLRC4 (Inflammosome),

- PGLYRP1 (Bakterizidie Gram-positive Keime),

- MMP9 (Wundheilung),

- DEFA4 (Membrandestruktion),

- SLPI (Leukozyteneptidase-Inhibitor),

- CAMP (Cathelicidin).

Verglichen mit dem initialen Befund nahm die Genexpression bei den Patienten in 12 Monaten insgesamt zu. Dies galt bei stabilen und progressiven Verläufen. Die absolute Expressionssteigerung korrelierte mit dem Überleben. Im Unterschied zu nicht progredienten Fällen hatten Patienten mit einer Krankheitsverschlechterung nach 1, 3, 6 und 12 Monaten signifikant höhere Absolutwerte für MMP9 und SLPI ( $p=0,05$ und $p$ $=0,008)$.

FAZIT

Patienten mit IPF hatten ein verändertes respiratorisches Mikrobiom und eine spezifisch antimikrobielle Abwehrsteigerung, die mit dem Verlauf korrelierte und kontinuierlich nachweisbar war. Diesen Dauerstress durch das veränderte und/oder verstärkte Mikrobiom interpretieren die Autoren als Stimulus für die wiederholte Alveolarschädigung. Interventionsstudien mit Antibiotika oder anderen Mikrobiomnachweisen könnten die klinische Relevanz der Ergebnisse überprüfen.

Dr. med. Susanne Krome, Melle 